\title{
Polarization of Variable Stars
}

\section{Introduction}

\author{
K. SERKOWSKI (Tucson)
}

The changes in stellar polarization with time were first discovered in 1958 by GRIGORYAN (1958) for the red supergiant $\mu$ Cephei. In subsequent year BEHR (1959) gave a hint in a footnote to his catalogue that the emission-line B-type star $\gamma$ Cas seems to have variable polarization. However, several years had to pass before these discoveries were confirmed and the changes in polarization with time for the red giants and supergiants and for the emissionline B-type stars were firmly established, first by the observations made by SHAKHOVSKOJ $(1963,1964)$ and then by COYNE, GEHRELS $(1967)$ and the writer (SERKOWSKI 1966, 1968). The changes in polarization with time were also found for the RV Tauri and T Tauri stars, $R$ Coronae Borealis stars, novae, Wolf-Rayet stars, and white dwarfs. The rapid changes in polarization with time indicate that the polarization is not produced in the interstellar medium but in the vicinity of a star; such polarization is called intrinsic.

Despite great diversity of stars exhibiting the intrinsic polarization all of them, except the white dwarfs, seem to have a common feature: every time when a star with intrinsic polarization was observed in the infrared, at $10 \mu$ or $11 \mu$ wavelength, a pronounced excess of infrared radiation was found, as compared to that of unpolarized stars of similar spectral type. For the emission-line B-type stars the infrared excess was explained by WOOLF, STEIN and STRITTMATTER (1970) as a thermal free-free radiation of the hot ionized gas envelope surrounding the star. The spectral emission lines are produced in the same envelope. The free-free emission contributes also to the infrared excess observed in the late-type stars with the emission lines in their spectra. However, for majority of intrinsically polarized late-type stars the spectral energy distribution in the infrared indicates the thermal emission of a black body with temperature of about $600^{\circ} \mathrm{K}$ superimposed upon the stellar emission (NEUGEBAUER, BECKLIN and HYLAND 1971). This is explained as the thermal emission of dust envelope surrounding the star.

WALLERSTEIN (1971) suggested recently that the envelope emission from stars with an infrared excess probably comes both from dust and from free-free emission in varying degrees, depending upon the ratio of dust to gas and the level of excitation of the gas. The most extreme dusty object is NML Cyg which does not show any emission lines at all. Most latetype intrinsically polarized stars show both the emission-lines in spectrum and the dust

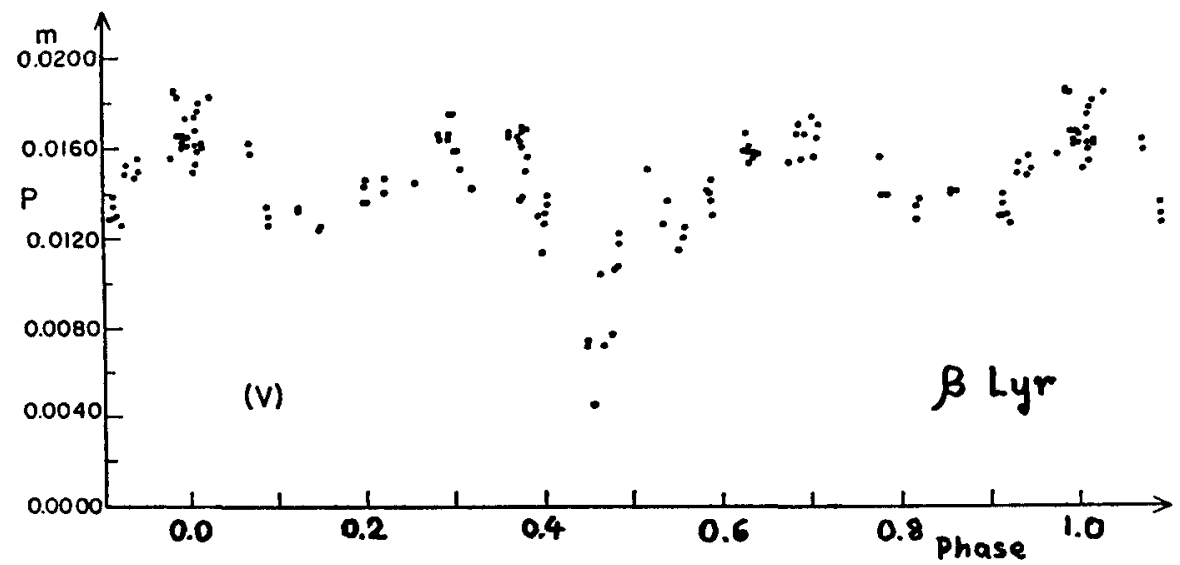

Fig. 1: Polarization of $\beta$ Lyrae in yellow spectral region, according to APPENZELLER and HILTNER (1965). Polarization is expressed in magnitudes and plotted against phase. 


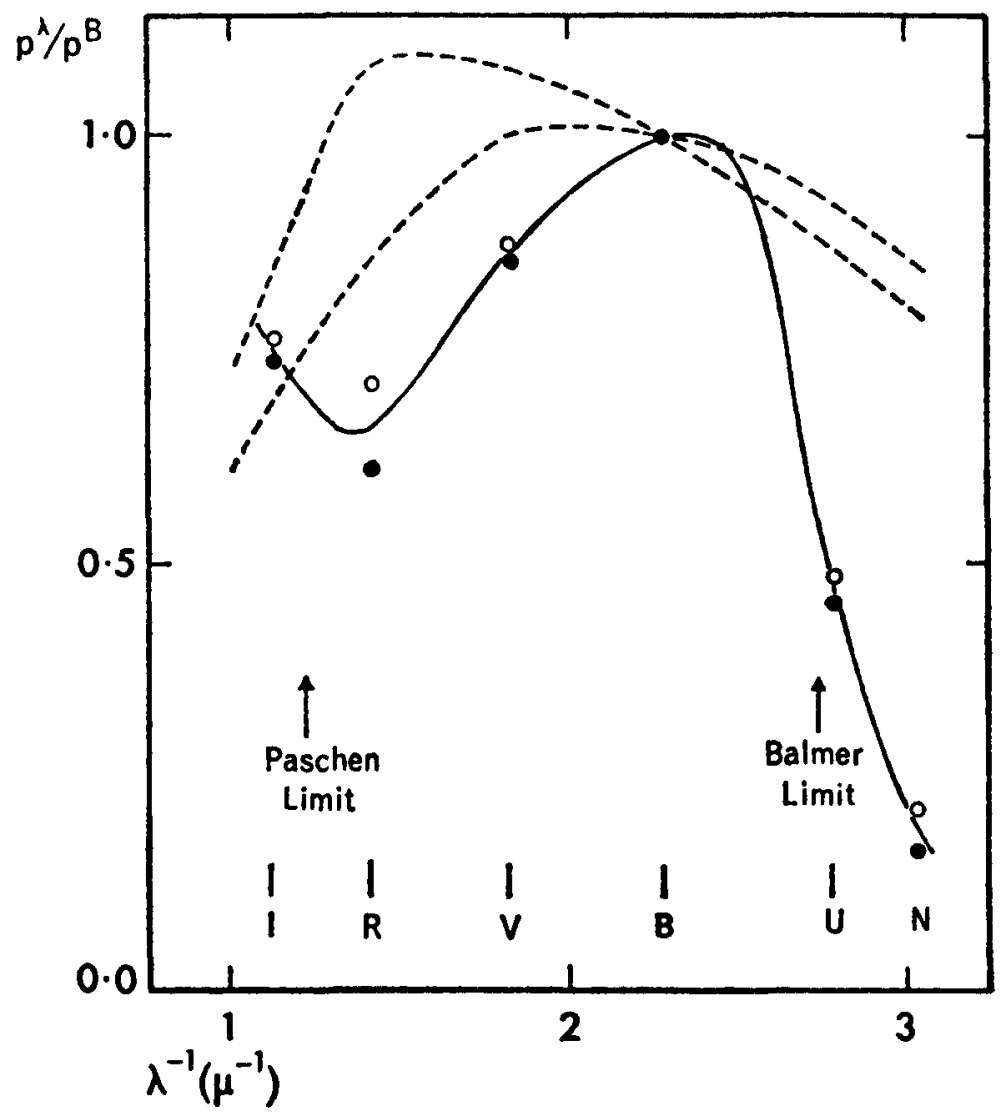

Fig. 2: Wavelength dependence of intrinsic polarization of Be stars p Car (filled circles) and $\alpha$ Ara (open circles) observed by SERKOWSKI (1970a) compared with that of interstellar polarization for two cases: $\mathrm{P}^{\mathrm{V}} / \mathrm{P}^{\mathrm{B}}=1.0$ and 1.1 (dashed lines).

envelope. The emission-line B-type stars represent probably the extreme situation of free-free emission only.

I shall first discuss the intrinsic polarization of the early-type stars, proceeding then to the later spectral types.

\section{Intrinsic Polarization of Early-Type Stars}

The first early-type star for which the changes in polarization with time were studied in detail is the supergiant eclipsing binary $\beta$ Lyrae for which such changes were detected by SHAKHOVSKOJ at Crimean Observatory in 1962. SHAKHOVSKOJ $(1962,1964)$ explained the variable component of polarization as produced by scattering of light from electrons in the disk-shaped envelope surrounding the binary; the light scattered at an angle of $90^{\circ}$ may be 100 per cent linearly polarized. The secondary maxima of the polarization curve (Fig. 1 ) were explained by RUCINSKI $(1966,1967)$ as an effect of the reflection of light of the primary by the secondary component.

First study of the wavelength dependence of the intrinsic polarization of $\beta$ Lyrae was made by APPENZELLER and HILTNER (1967); it was extended last year by COYNE (1970a) to 
wider spectral range, from 3300 to 9600 A. APPENZELLER and HILTNER found that polarization in the ultraviolet is considerably smaller than in the blue and yellow spectral regions - the same effect which I found a year later (SERKOWSKI 1968, 1970a) for the intrinsic polarization of non-binary emission-line B-type stars (Fig. 2). APPENZELLER and HILTNER explained this wavelength dependence by absorption of starlight by hydrogen before and after scattering from electrons in the stellar envelope; the same explanation holds for the intrinsic polarization of emission-line B-type stars which are not binaries. For such stars the minima in the wavelength dependence of polarization are observed on the short wavelength side of the limits of Balmer and Paschen series.

The changes in polarization during the eclipse were discovered by SHAKHOVSKOJ (1964, 1969a) and by SHULOV (1967) for about 12 other eclipsing binaries. For most of these stars the spectra indicate the presence of gas streams or rings. The most prominent examples of such stars among these with variable polarization are the Wolf-Rayet binary V 444 Cygni (SHULOV 1966, HILTNER and MOOK 1966), and the binaries U Sge and RY Per (Fig. 3); for this latter star the changes in polarization with time were suspected already in 1947 by HILTNER (1947) and then studied by SHAKHOVSKOJ (1964) and by SHULOV and GUDKOVA (1969). For peculiar Ae-type hydrogen deficient eclipsing binary $v$ Sgr the changes in polarization during the primary eclipse were noticed by COYNE and GEHRELS (1967) and studied recently in somewhat more detail by COYNE and KRUSZEWSKI (1971).

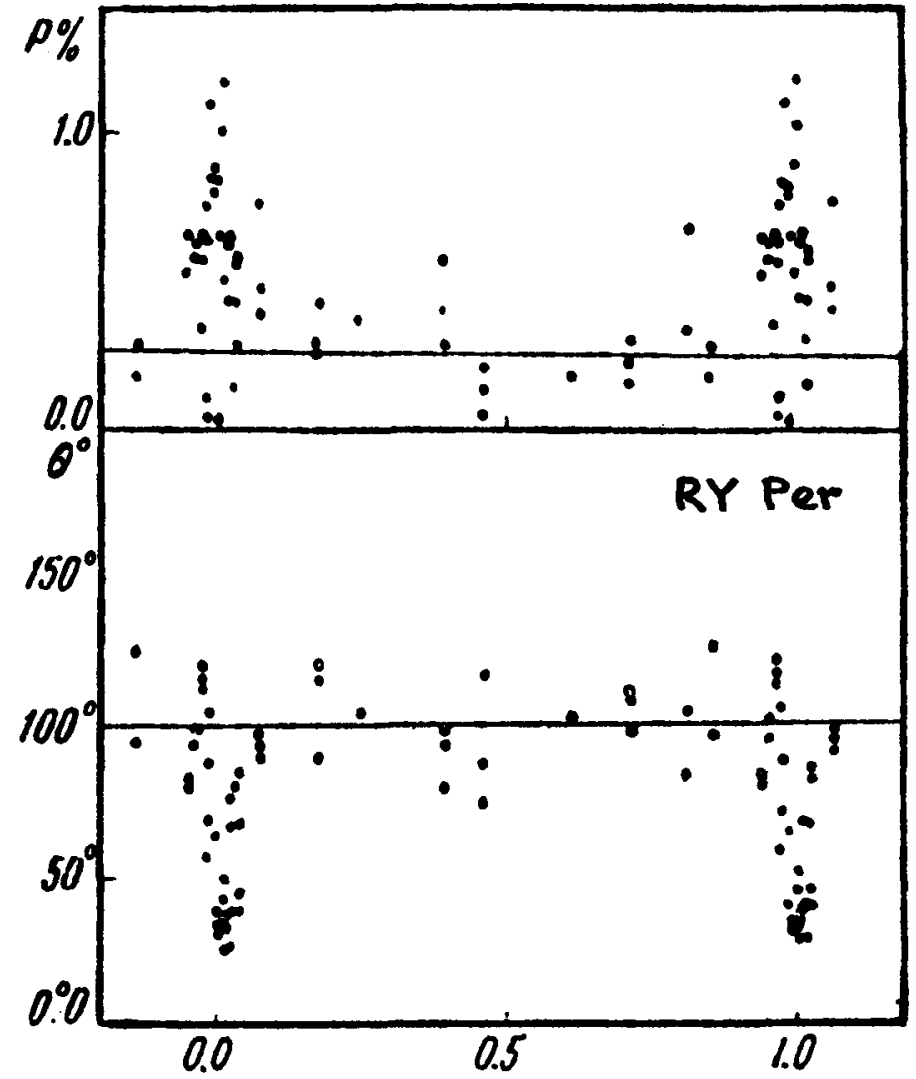

Fig. 3: Intrinsic percentage polarization and position angles of eclipsing binary RY Per according to SHULOV and GUDKOVA (1969), plotted against phase. 


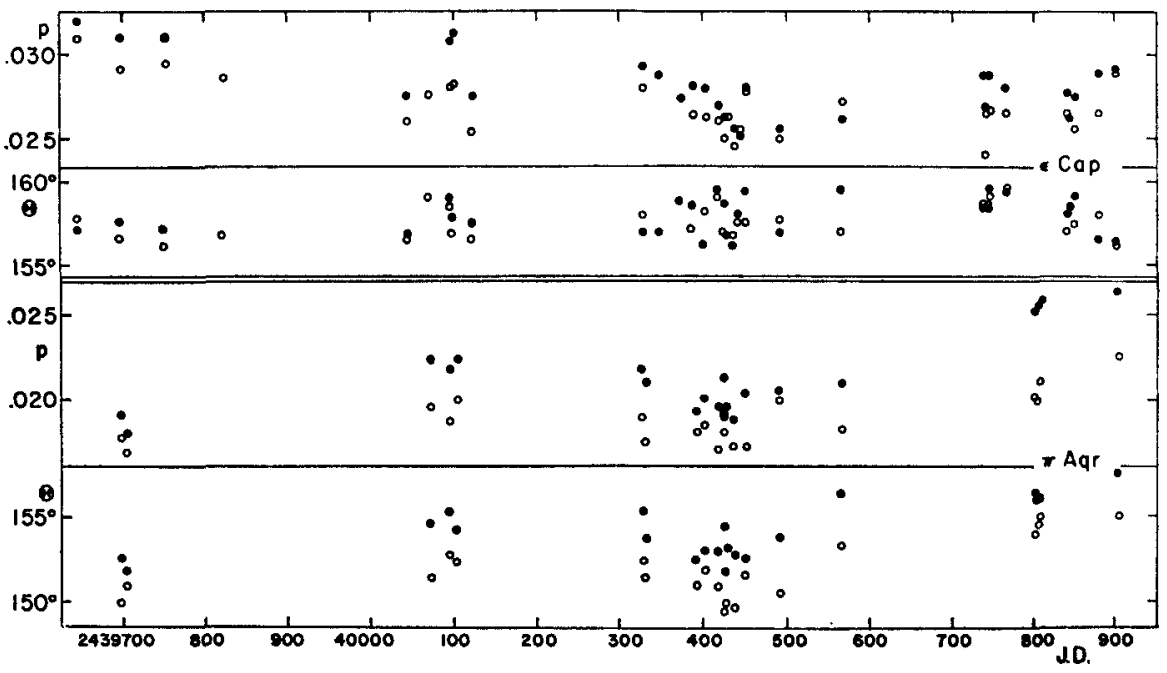

Fig. 4: Changes in polarization of Be stars $\varepsilon$ Cap and $\pi$ Aqr in the blue (filled circles) spectral regions, according to STRITTMATTER and SERKOWSKI (1971).

I have tried to detect changes in polarization with time for about 14 among the brightest southern eclipsing binaries (SERKOWSKI 1970a). As a result of this study $I$ found changes in polarization with time for only two of these stars: TT Hya (A3e + dG6p) and V453 Sco $(\mathrm{B} 1 \mathrm{Iab}+\mathrm{Be})$. For TT Hya polarization increases by $0.75 \%$ at primary eclipse when the emission lines of hydrogen are visible in the spectrum of G-type component. V453 Scorpii has a Be-type component. My conclusion from this study was that only the eclipsing binaries with emission lines in their spectra would show changes in polarization with time; $\beta$ Lyrae remains the only binary for which this polarization was studied in some detail. The prediction of large polarization effects in early-type eclipsing binaries by CHANDRASEKHAR in 1946, which led to the discovery of interstellar polarization, was based on grey atmosphere approximation. Similar calculations for non-grey atmospheres of early-type stars were made by NAGIRNER (1962) at Leningrad Observatory. His calculations indicated polarization at least order of magnitude smaller than that predicted by CHANDRASEKHAR for grey atmospheres. NAGIRNER's results were confirmed by more accurate calculations made last year by COLLINS (1970) and by RUCIŃSKI (1970). According to COLLINS for $\lambda>3000 \mathrm{~A}$ the largest polarization on the disk of a rotationally distorted early-type star is observed at its pole where polarization reaches the value of 0.5 per cent, 20 times less than for the grey atmosphere. COLLINS predicts large polarization only in the far ultraviolet, around the Lyman limit of hydrogen. Therefore the observed polarization should be attributed not to stellar atmospheres but to the extended envelopes.

The changes in polarization with time observed for non-binary Be stars (Fig. 4, from STRITTMATTER and SERKOWSKI 1971) are irregular; the rapid variations with time scale of a few days are superimposed upon the slow changes with time scale of years. The star with the largest amplitude of such slow changes in polarization is the nova-like shell star Z CMa (SERKOWSKI 1970a). Nobody searched for variations with time scale of a few minutes which are likely for Bestars since such rapid variations in the equivalent widths of hydrogen emission lines were detected by BAHNG (1971) for $\zeta$ Tauri.

The irregular changes in polarization are observed for at least 10 per cent of a sample of early-type supergiants (SERKOWSKI 1970a, COYNE 1971). This is troublesome for the 


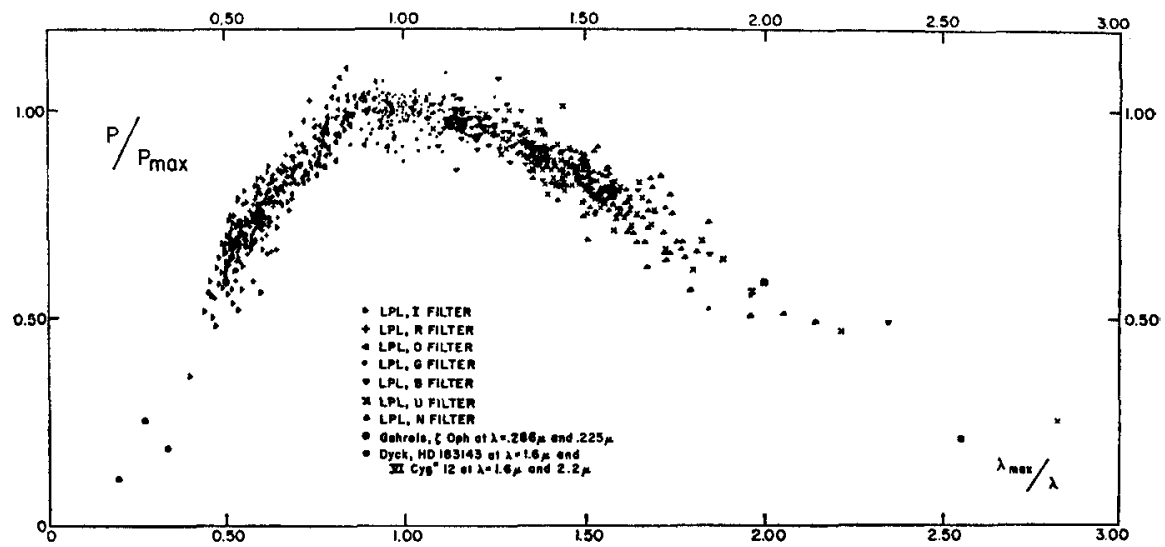

Fig. 5: Wavelength dependence of interstellar polarization according to the observations made by G.V. COYNE and T. GEHRELS at the Lunar and Planetary Laboratory. The squares denote the ultraviolet observations of $\zeta$ Oph made from balloon by GEHRELS. The open circles are the unpublished DYCK's infrared observations.

studies of the interstellar polarization which are based mostly on such supergiants. Some help in distinguishing the intrinsic from the interstellar polarization is obtained from the fact that the wavelength dependence of interstellar polarization (Fig. 5) was found recently by the writer to follow closely the formula

$$
\mathrm{P}(\lambda) / \mathrm{P}\left(\lambda_{\max }\right)=\left(\lambda_{\max } / \lambda\right)-1.09 \ln \left(\lambda_{\max } / \lambda\right)
$$

where $\lambda_{\max }$ is the wavelength $\lambda$ at which the degree of polarization $\mathrm{P}(\lambda)$ of any particular star takes maximum value; $\lambda_{\max }$ takes values from $0.45 \mu$ to $1.0 \mu$. Because of variability of the polarization of many supergiants the difficulties are encountered in finding the highly polarized standard stars with constant polarization; such stars are needed for comparing the different series of polarimetric observations. The best choice seems to be either the early-type supergiants without the infrared excess, e. g. HD 183143 (GEHRZ, WOOLF 1971), or the mainsequence B-type stars with large interstellar polarization (e. g. HD 154445) or, finally, the classical cepheids, as for none of them the changes in polarization were detected.

The peculiar irregular changes in polarization with time were found for the Wolf-Rayet star HD 50896 (SERKOWSKI 1970a); the polarization is larger in the ultraviolet than in the blue and yellow spectral regions which is unique among the early-type stars.

An increase in polarization of the recurrent nova $T$ Pyx in the blue and yellow spectral regions was observed during its outburst in 1966 (EGGEN, MATHEWSON and SERKOWSK1 1967). The polarization in the ultraviolet spectral region changed very little and remained during the outburst almost 3 times smaller than one in the blue. Similar behaviour was observed for Nova Delphini 1967 (ZELLNER and MORRISON 1971). During the first 120 days after the outburst the intrinsic polarization was sharply peaked over a wavelength with a maximum averaging $0.6 \%$ in red light. The intrinsic polarization in the ultraviolet was less than half of that in the red. A strong infrared excess was observed for this nova by GEISEL, KLEINMANN and LOW (1970) who attributed it to thermal radiation by circumstellar grains. However, ZELLNER (1971) suggests that different dust grains are responsible for the optical polarization, smaller than those producing the infrared excess, which appeared at a later time. For the peculiar object Eta Carinae, for which large polarization was discovered by THACKERAY (1956) the polarization in the optical region remains almost independent of the wavelength (VISVANATHAN 1967). 
The studies of the changes in polarization with wavelength across the spectral lines are a wide field of research which I shall not be discussing in detail. Such studies of circular polarization are, of course, the basis of the measurements of stellar magnetic fields initiated by BABCOCK (1947). Similar studies of the changes in I in e a r polarization across the hydrogen lines of $\beta$ Lyrae were made already in 1934 by OHMAN (1934) in Stockholm Observatory. He found faint polarization effects in the absorption contour of $\mathrm{H} \gamma$. Similar effects for other early-type stars were found by CLARKE and GRAINGER (1966). Further study of such effects seems to be very urgent.

The numerous attempts to detect the changes in the wide-band linear polarization of magnetic stars (e.g. THIESSEN 1961, ELVIUS and ENGBERG 1967, HILTNER and MOOK 1967, SERKOWSKI and CHOJNACKI 1969, POLOSUKHINA 1969, COYNE 1970b) did not prove the existence of such changes. Recently, however, the wide-band intrinsic circular and linear polarization produced by the gigantic magnetic fields of peculiar white dwarfs Grw $+70^{\circ} 8247$ (Figs. 6, 7), G195-19 and G99-37, were detected by KEMP, SWENDLUND, LANDSTREET and ANGEL (1970) by ANGEL and LANDSTREET (1971) and by LAND-

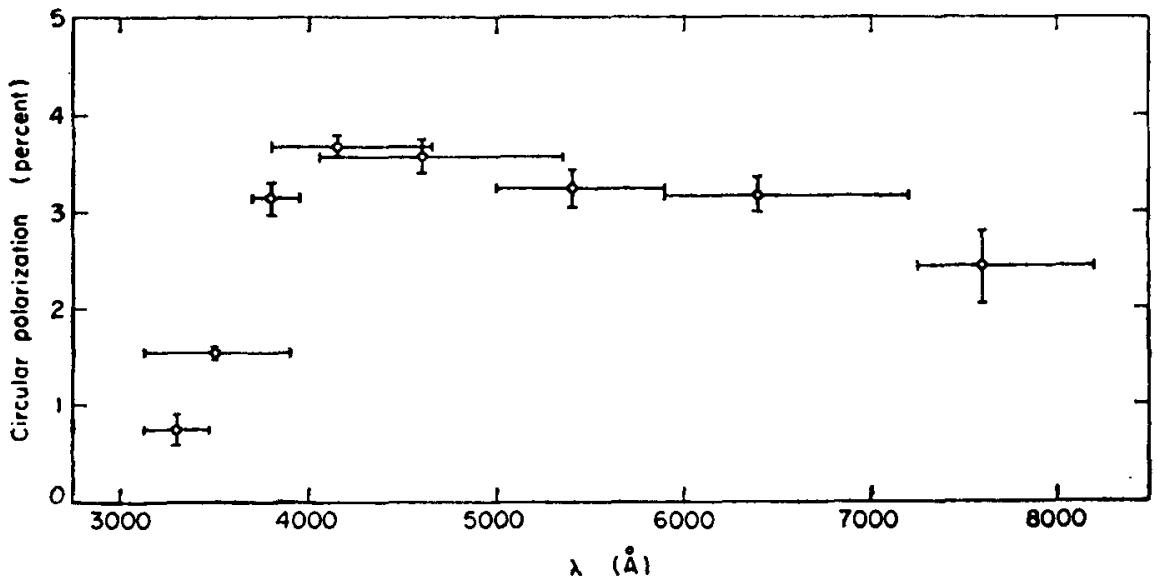

Fig. 6: The wavelength dependence of the circular polarization of white dwarf $\mathrm{Grw}+70^{\circ} 8246$; a combination of the observations of ANGEL and LANDSTREET (1970) and the unpublished observations by GEHRELS (cf. GEHRELS 1971). The vertical bars indicate the probable errors. A 15 percent circular polarization at $1.25 \mu$ wavelength was detected by KEMP and SWENDLUND (1970).

STREET and ANGEL (1971), respectively. These discoveries resulted from predictions of the theory of gray body magnetoemission developed by KEMP (1970). The magnetic field is on the order of $10^{7}$ gauss. For G195-19 the polarization is changing with a period of 1.34 days. Large changes in polarization were also found for the Crab Nebula Pulsar.

\section{Intrinsic Polarization of Late-Type Stars}

An increase in polarization from 0.5 to at least 1.3 per cent was observed during the deep minimum of the R Coronae Borealis star RY Sgr (SERKOWSKI and KRUSZEWSKI 1969); this was accompanied by a large change in the position angle of polarization. Another group of stars with intrinsic polarization are the $T$ Tauri stars. For the $T$ Tauri itself the chaotic changes in polarization with time were discovered by VARDANIAN (1964) and confirmed by the writer (1969a, 1971). Unfortunately, very little is known yet about the wavelength dependence of its intrinsic polarization. For another $\mathrm{T}$ Tauri star $\mathrm{R}$ Mon and for the cometary 


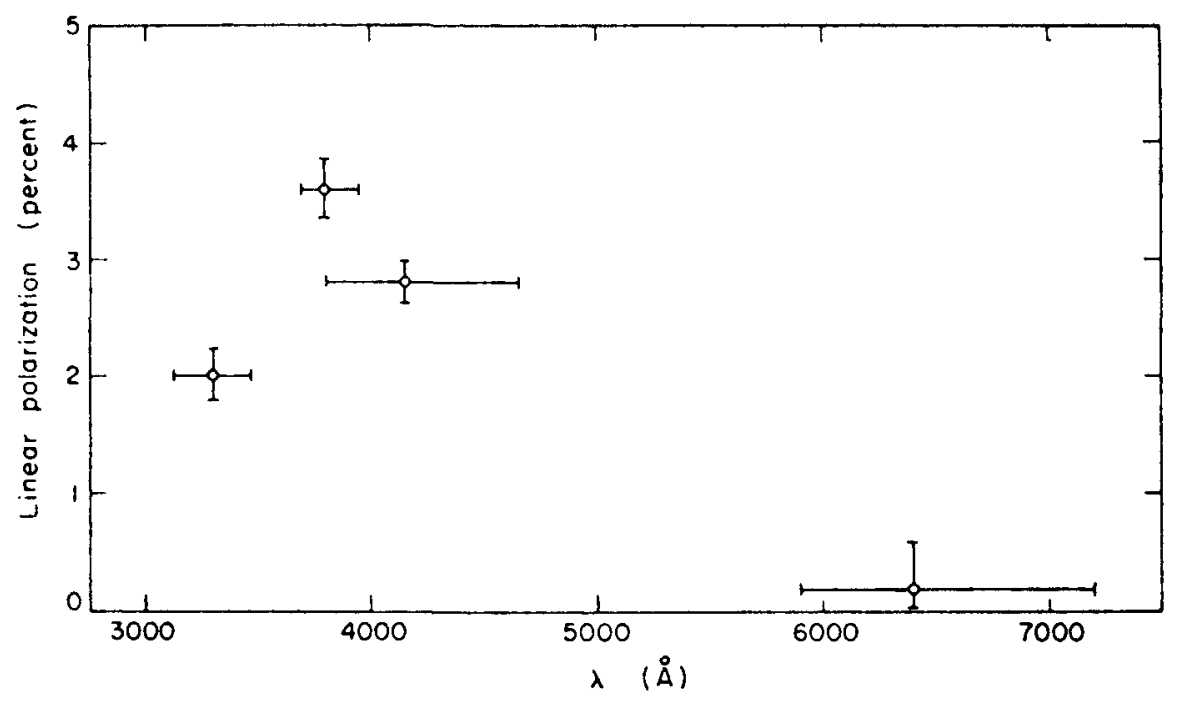

Fig. 7: Same as Fig. 6 for linear polarization.

reflection nebula surrounding this star, large polarization was found by R. HALL (1965) and studied subsequently by ZELLNER (1970) who found that the variable polarization, which usually exceeds 10 per cent, is almost independent of wavelength. According to ZELLNER the lack of increase in polarization towards ultraviolet rules out Rayleigh scattering as a source of polarization but may be compatible with scattering by relatively large dust grains. The writer found rapid changes in polarization for $\mathrm{R} \mathrm{CrA}$ which is an object very similar to $\mathrm{R}$ Mon (SERKOWSKI 1969b). For these objects it seems that it is only the light of reflection nebula which is polarized, and not the direct starlight.

There were several attempts to detect the intrinsic polarization of flare stars during the flare. The recent observations of YZ CMi and UV Cet by VARDANIAN (1969) indicate that the polarization during flare, if any, does not exceed 0.8 per cent.

The changes in polarization of the RV Tauri stars $R$ Sct and $U$ Mon were found by SHAKHOVSKOJ (1963) and ALIEV (1965). For R Sct SHAKHOVSKOJ (1969b) noticed that the intrinsic polarization in $U, B$ and V spectral regions is increasing steeply towards the ultraviolet, following approximately the Rayleigh's $\lambda^{-4}$ law. The wavelength dependence of polarization is less steep for U Mon as can be seen on the radial graphs (Fig. 8) used by the writer for subtracting the interstellar polarization. Of the two stars $\mathrm{U}$ Mon shows much more regular changes both in brightness and in polarization. Most striking is the dependence of the position angle of the intrinsic polarization of U Mon on the phase of light variations (Fig. 9, SERKOWSKI 1970b). It remains close to $180^{\circ}$ before and around the deep light minima and close to $105^{\circ}$ before and around the shallow light minima that occur halfway between the deep minima. The position angle changes abruptly by $75^{\circ}$ at the time when doubling of the spectral lines is observed, about 14 days after each light minimum.

The large infrared excess at the wavelength of $10 \mu$ was found for U Mon by GEHRZ and WOOLF (1970). If both the infrared excess and intrinsic polarization are produced by dust envelope the changes in position angle can be explained if the shell of gas and dust is ejected during the deep light minima preponderantly along one, and during the shallow minima along another of the two perpendicular diameters of the star. The fact that the observed change in position angle is by $75^{\circ}$ and not by $90^{\circ}$ can be explained by perspective effects. 


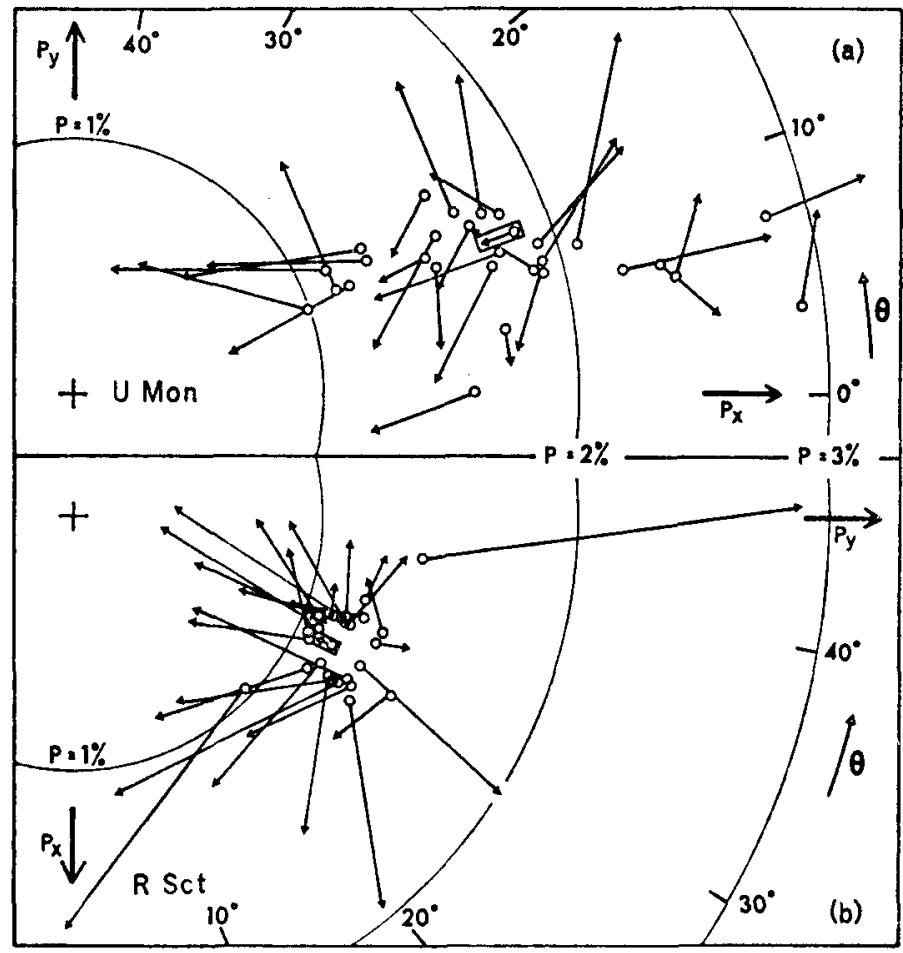

Fig. 8: Polarization of RV Tauri stars U Mon (a) and R Sct (b) observed in the yellow (open circles) and the ultraviolet (tips of arrows) by SERKOWSKI (1970b), plotted in radial coordinates. Distance of each symbol from origin of the coordinates (cross) is proportional to the percentage polarization, $\mathrm{P}$; the angular coordinate is the double position angle, $2 \Theta$. Figure indicates that the majority of arrows are pointing outward from the arrow surrounded by a rectangle, which represents the interstellar component of polarization.

Because of similarities between the RV Tauri and W Virginis stars the intrinsic polarization may be expected for the latter stars too. I made a number of polarimetric observations of $\mathrm{W}$ Virginis which seem to indicate the dhanges in polarization with time. The number of the observations is still insufficient for deciding whether these changes in polarization repeat with the same period as the brightness and radial velocity changes.

The early polarimetric observations of red variable stars by GRIGORYAN (1958), SHAKHOVSKOJ (1963) and other Russian observers were limited to the objects situated close to the galactic equator and the wavelength dependence of polarization was not studied. The intrinsic polarization was mixed with the interstellar polarization and little could be said about its properties. Only in 1966 large polarization, increasing steeply towards the ultraviolet, was found for several high galactic latitude miras and semiregular variables, some of them carbon stars (SERKOWSKI 1966). The changes in polarization with time for some of these stars are shown in Figs. 10 and 11. For V CVn, a M4IIIe star with 198 days period of light variations, the largest polarization is observed at minimum light. For the miras of longer period, as o Ceti (see Fig. 2 of SERKOWSKI 1971) or R Hya (Fig. 11) the largest polarization is usually observed halfway between the minimum and maximum brightness. The wavelength dependence of position angles and its changes with time make the straightforward explanation 
impossible and indicate that at different wavelengths the different regions of stellar envelope are responsible for producing the polarization. The complicated character of these phenomena is indicated e. $\mathrm{g}$. by the $1970-71$ polarimetry of M7e mira variable $\mathrm{R}$ Leo plotted on radial diagram in Fig. 12.

The wavelength dependence of intrinsic polarization for every sufficiently well-studied red variable star dhanges its shape with time. However, for many miras and semiregular variable stars this wavelength dependence often follows the Rayleigh's $\hat{\lambda}^{-4}$ law (cf. Fig. 5 of SERKOWSKI 1971). This is also true for the carbon stars; the most notable example is IRC + 10216 (Fig.13), a source of CO radio emission, for which SHAWL and ZELLNER (1970) found 20 per cent polarization at $1 \mu$. Because of its extreme redness this carbon star could not be observed at wavelengths shorter than $0.6 \mu$.

The wavelength dependence of polarization proportional to $\lambda^{-4}$ suggested the Rayleigh scattering on gas molecules in the stellar atmosphere as a mechanism producing the polarization. This mechanism, first suggested by SHAKHOVSKOJ (1963), was discussed by KRUSZEWSKI et al. (1968) and then by HARRINGTON (1969, 1970) who indicated that the observed large polarization can be explained by Rayleigh scattering on molecular hydrogen if only there is a large gradient of source function, appreciable absorption, e. g. by negative hydrogen ions, and a few hundred degrees difference in temperature between the pole and the equator of the star. This mechanism may be very attractive for explaining the previously discussed changes in polarization of $U$ Mon if the star undergoes the non-radial oscillations. The difficulties with applying this mechanism to M-type stars were pointed out by DYCK and SANDFORD (1971): If the molecular scattering does not occur in the envelope but in the stellar atmosphere the wavelength dependence of polarization must have the extrema at

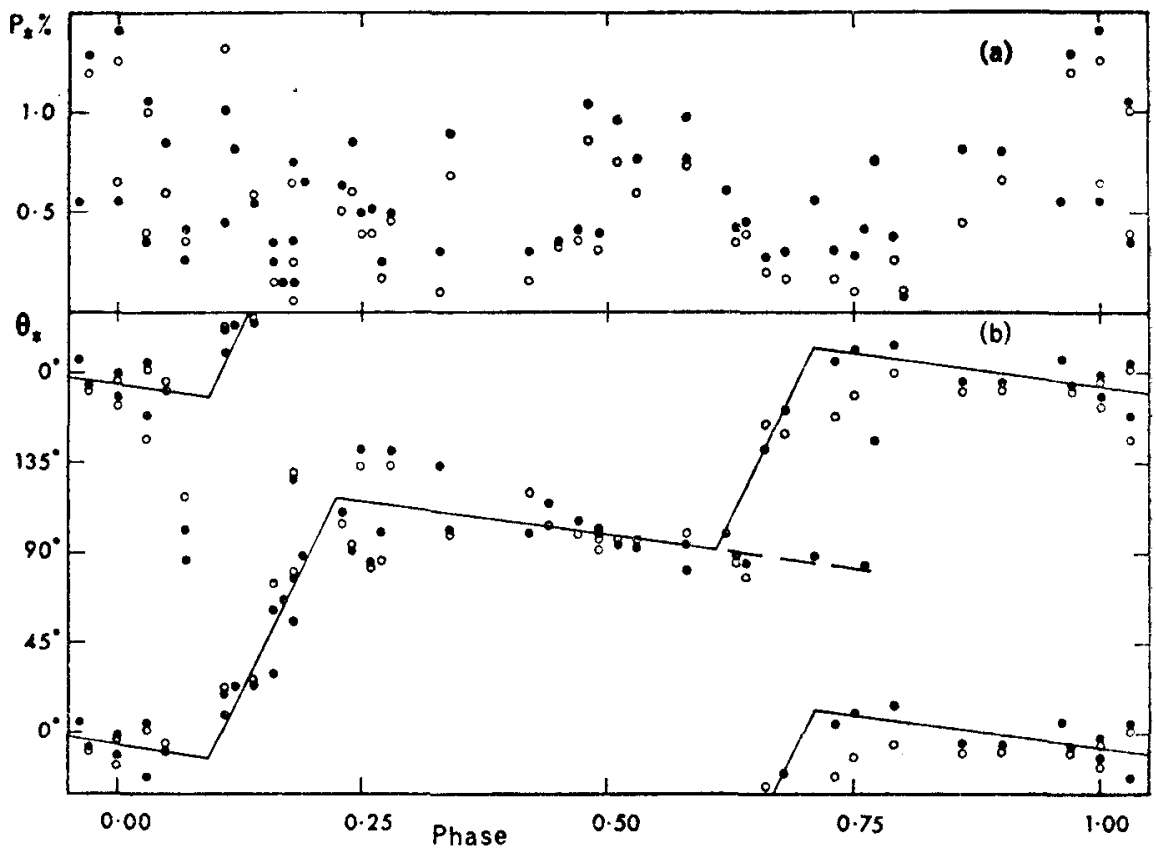

Fig. 9: Changes in the intrinsic component of the polarization of RV Tauri star U Mon, obscrved by SERKOWSKI (1970b) in the yellow (open circles) and the blue (filled circles) spectral regions, with the phase of light variations. (a) Percentage polarization, (b) Position angle. 


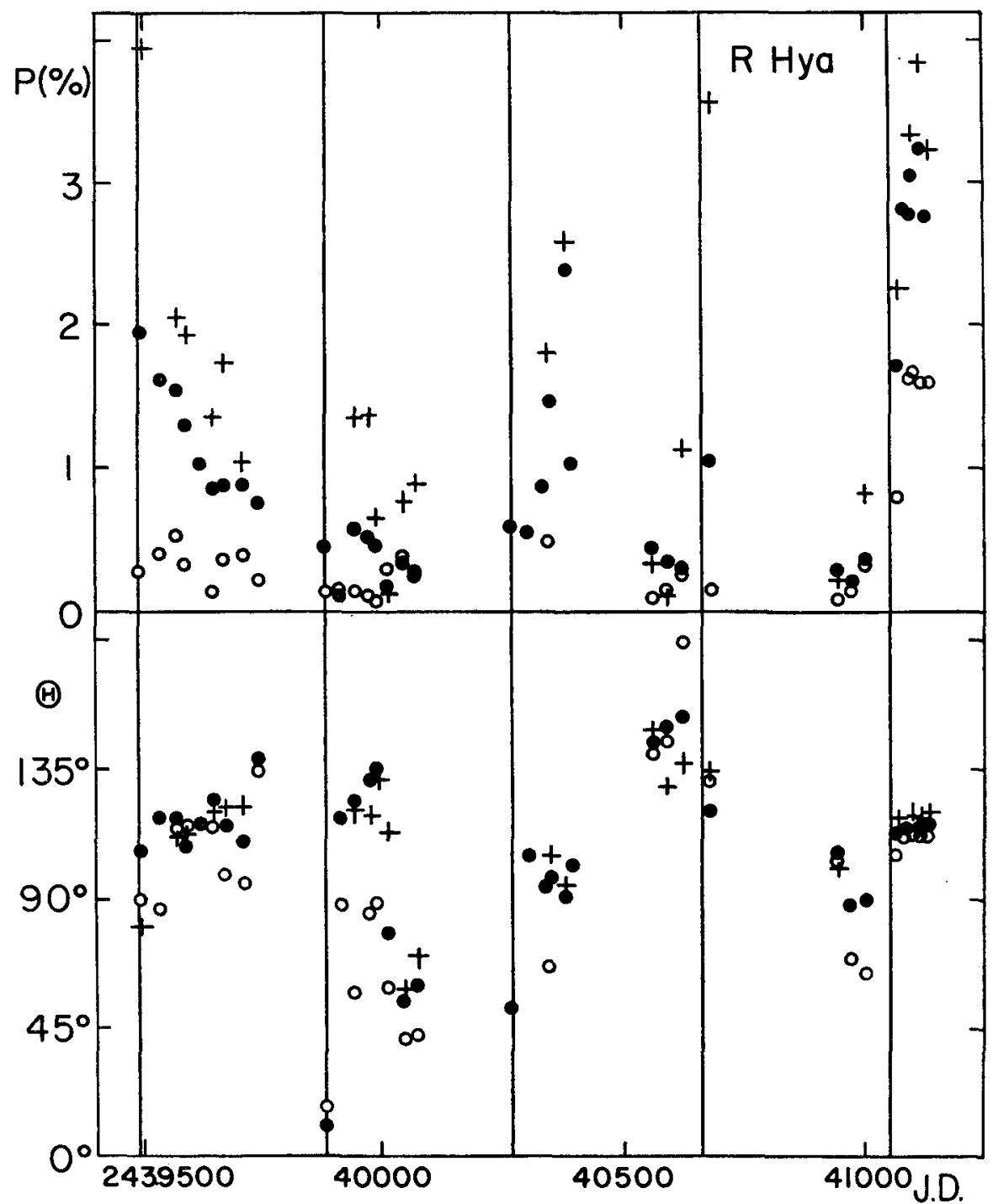

Fig.10: Percentage polarization and position angle for the mira variable $\mathrm{R}$ Hya in the yellow (open circles), blue (filled circles) and ultraviolet (crosses) spectral regions. Vertical lines indicate the times of minimum light.

$\mathrm{TiO}$ bands which were not found in the high spectral resolution polarimetry by DOMBROVSKIJ (1970). However, these high resolution observations are not very accurate and should be repeated; the UBV bands are not wide enough to detect the effects of $\mathrm{TiO}$ bands on the wavelength dependence of polarization even if these effects were large. DYCK and JENNINGS (1971) point out that there is no polarization and no infrared excess for the stars $\eta$ Gem, $\pi$ Aur and $\alpha$ Her for which DEUTSCH (1960) found expanding circumstellar gas; this also is an argument against the polarization produced by the molecular scattering in the stellar envelope. 


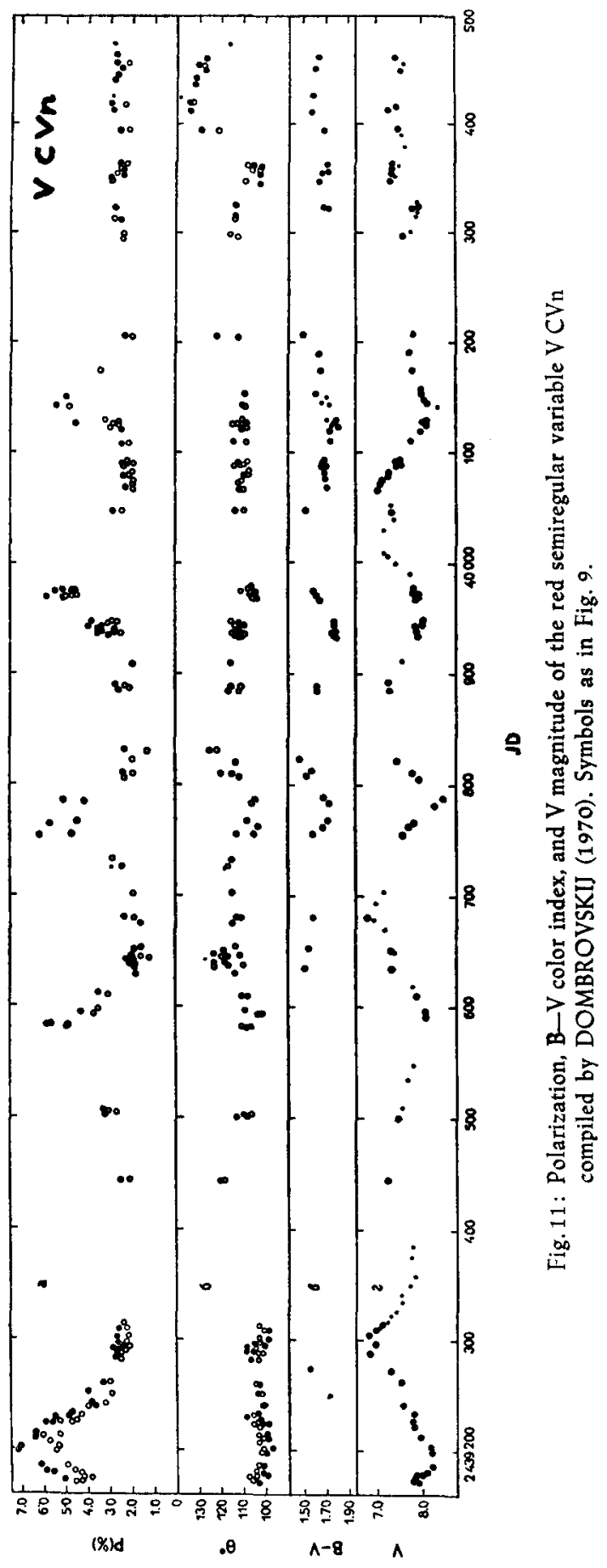




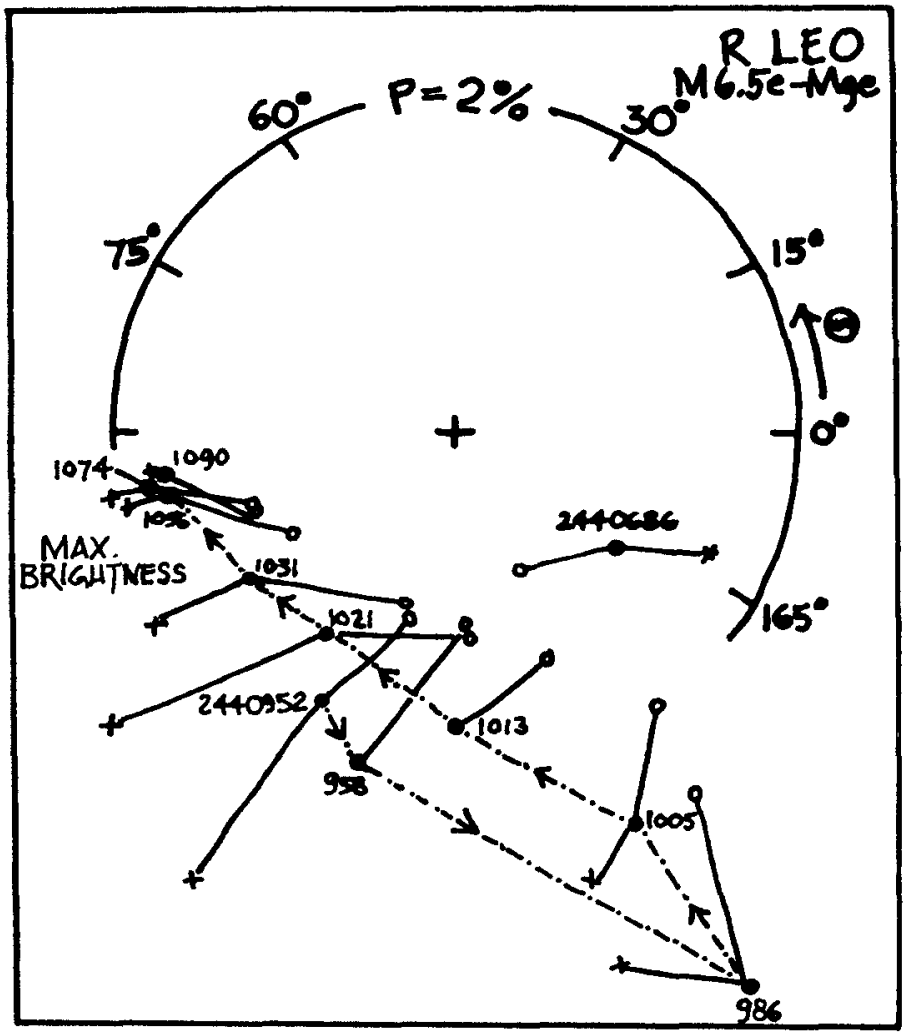

Fig. 12: Polarization of mira variable R Leo plotted in radial coordinates. Full lines join the simultaneous observations in the yellow (open circles), blue (filled circles) and ultraviolet (crosses) spectral regions. The dashed line joins the observations in the blue spectral region made on different nights. The numbers are the Julian Day numbers.

The correlation between the polarization and infrared excess (Fig. 14) found by DYCK et al. (1971) is a strong argument in favour of scattering on the circumstellar dust grains as a mechanism producing the intrinsic polarization. Similar explanation was proposed by DONN et al. (1966) who, however, suggested that the intrinsic polarization of red variable stars is produced by the forward scattering of stellar light by graphite platelets aligned by stellar magnetic field. Several authors were developing this hypothesis further (e. g. PENSTON 1967, K. H. SCHMIDT 1968, FRIEDEMANN 1970, SVATOS 1970, KRISHNA SWAMY 1970). In my opinion, assuming the alignment of grains by magnetic field is not necessary. The light scattered at $90^{\circ}$ scattering angle from non-aligned, isotropic, spherical dust grains may be almost 100 per cent polarized. Similarly high polarization of starlight would be observed if mainly the scattered light is seen and the direct starlight is obstructed by a dense dust cloud.

DYCK et al. (1971) used the relationship between polarization and infrared excess (Fig. 14) for estimating that the albedo of dust grains is larger than 0.5 . They noticed that this relationship holds both for $M$-type and carbon stars which they consider as a strong argument against polarization produced by the molecular scattering. The infrared excess of carbon stars has different character than that of M-type stars (WOOLF and NEY 1969); in case of R Lep it 


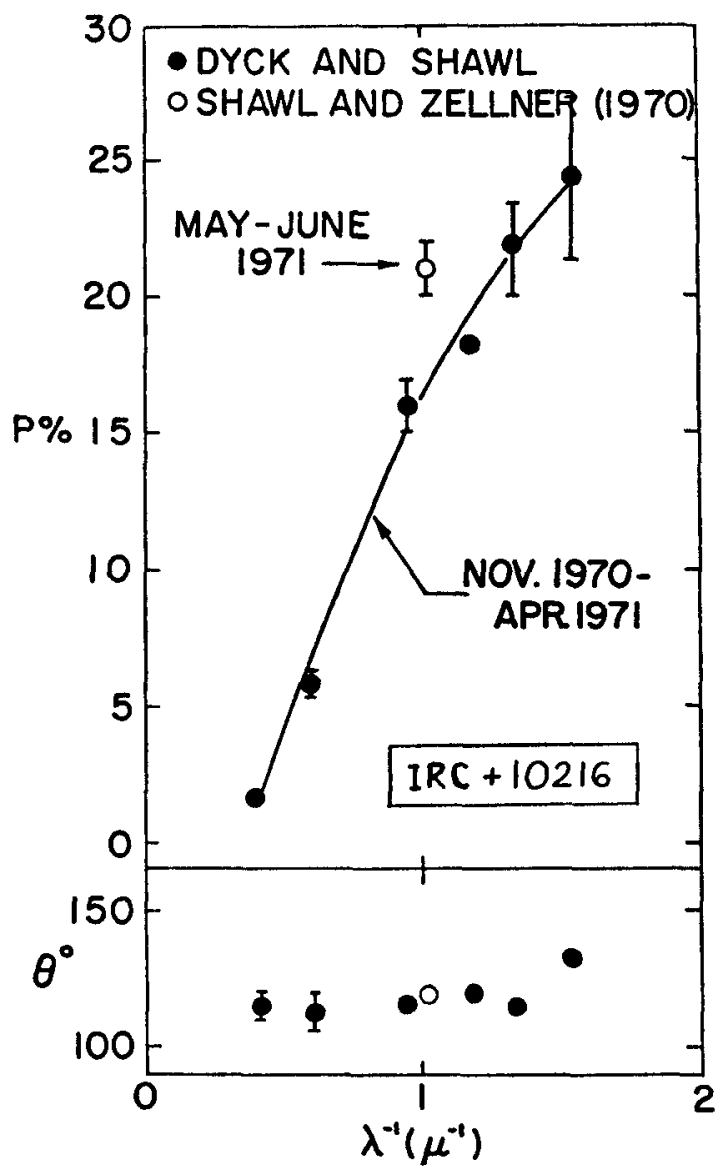

Fig. 13: The wavelength dependence of percentage polarization and position angle of IRC +10216 according to DYCK, FORBES and SHAWL (1971).

resembles the short wavelength end of $140^{\circ} \mathrm{K}$ blackbody emission while for the $M$-type stars the spectrum of infrared excess resembles the thermal emission of silicates. This is seen clearly on the infrared color-color diagram (Fig. 15) on which carbon stars are well separated from M-type stars (GILLETT, MERRILL and STEIN 1971). Two absorption features of silicates were found at $9.7 \mu$ and $10.6 \mu$ in the spectrum of M2Ib star 119 Tau (KNACKE et al. 1969). However, the wavelength dependence calculated by HANNER (1971) for the polarization of light scattered on spherical silicate grains does not seem to resemble the results of the observations; she suggests that grains of irregular shape may have quite different wavelength dependence of polarization.

DYCK and JENNINGS (1971) found negative correlation between the intrinsic polarization and Call emissions (reversals) in $M$-type stars other than the miras. They explain this correlation by cooling of chromosphere by the emission of the infrared radiation by dust. In miras the dust may be only above chromosphere which would explain the lack of correlation.

Several mira variables, e. $g$. R Dor and R Aqr seem to have a constant component of polarization in the ultraviolet spectral region which adds vectorially to the variable com- 
ponent of intrinsic polarization (cf. Figs. 8 and 9 of SERKOWSKI 1971). This may be connected with an ultraviolet excess observed in some miras, particularly around minimum light (MENDOZA 1967). VARDANIAN (1970) suggests synchrotron radiation as a source of this ultraviolet excess. It is interesting that similar pecularities in the behaviour of ultraviolet polarization are found for the M-type supergiant $\alpha$ Ori (Fig. 7 of SERKOWSKI 1971); the constant ultraviolet component seems to be oriented at position angle about $90^{\circ}$. One explanation of this component would be the influence of multiple scattering which is usually much stronger in the ultraviolet than at longer wavelengths.

The intrinsic polarization of late-type supergiants is difficult to study because most of them have large superimposed interstellar polarization (VARDANIAN 1966). The best studied among them is $\mu$ Cephei (GRIGORYAN 1958, 1970, COYNE and KRUSZEWSKI 1968) for

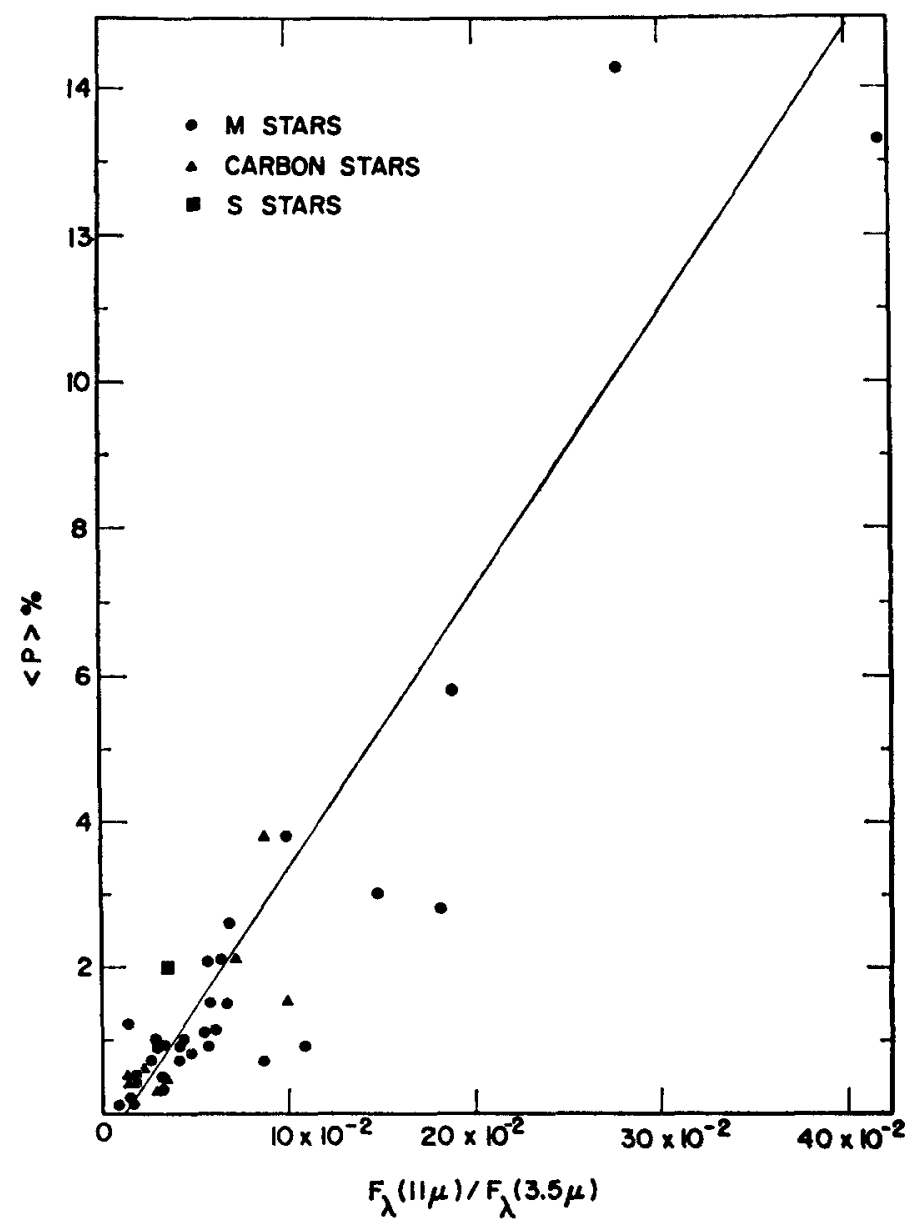

Fig. 14: Correlation between the mean percentage polarization of late-type stars in the blue and yellow spectral regions and the ratio of flur at $11 \mu$ to that at $3.5 \mu$, according to DYCK et al. (1971). 


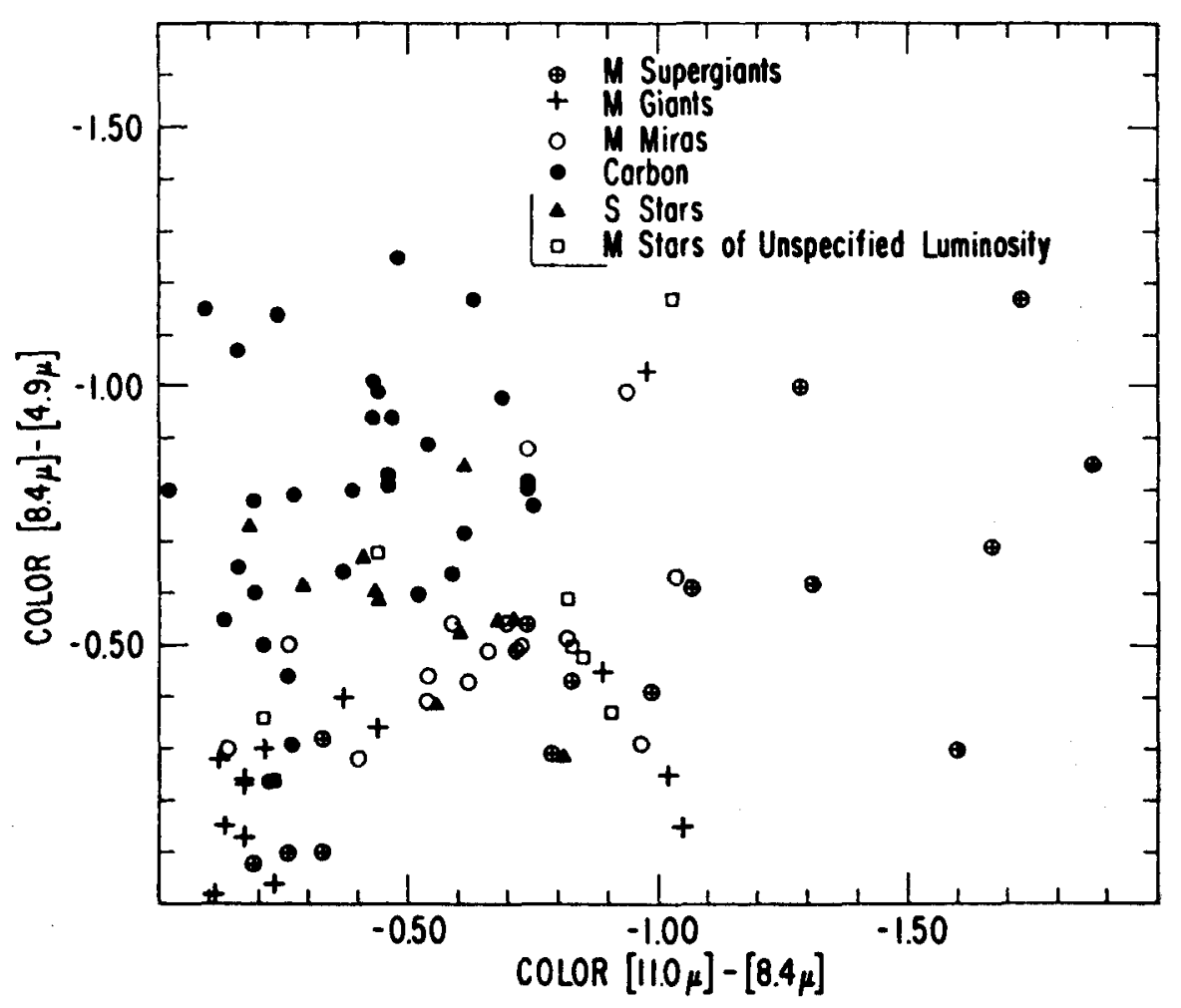

Fig. 15: Infrared two-color-index diagram for late-type stars, according to GILLETT, MERRILL and STEIN (1971) indicating difference between the infrared excesses of carbon stars and $M$-type stars.

which the polarization changes from 0.5 to 2.5 per cent but nevertheless determining and subtracting the interstellar component is not possible.

Undoubtedly the most interesting object among the M-type supergiants is VY Canis Majoris. Its large polarization, exceeding 20 per cent in the ultraviolet, was discovered by SHAWL (1969), and subsequently observed at wavelengths from $0.37 \mu$ to $3.5 \mu$ (FORBES 1971). The position angle of polarization depends very strongly on wavelength; on the radial diagram (Fig. 16) the simultaneous observations at different wavelengths are lying along the spiral line. Since the first infrared observations of VY CMa were made in January 1970 by HASHIMOTO et al. (1970) this spiral seems to be drifting slowly; another set of observations plotted in Fig. 16 are those made in January - March 1971 by DYCK, FORBES and SHAWL (1971). The infrared polarimetry made by FORBES (1971) in October 1970 and approximately simultaneous polarimetry at shorter wavelengths by the writer (SERKOWSKI 1971) falls between the two spiral lines in Fig. 16.

DYCK, FORBES and SHAWL (1971) explained the wavelength dependence of polarization of VY CMa by superposition of the interstellar polarization and the circumstellar polarization by silicate grains of the two sizes, $0.1 \mu$ and $1 \mu$ concentrated in different regions of the envelope. However, their assumption of somewhat improbably large interstellar component of polarization would not be needed if the polarization were produced in an elongated dust 
cloud in which the average size of dust grains would be decreasing with distance from the star; the long axis of this cloud would not be passing through the star. The changes in polarization with time would be explained by the motion of this cloud relative to the star. The outer regions of this dust cloud are visible as a reflection nebula for which polarization exceeding 40 percent was found (SERKOWSKI 1969b, 1971) in the blue and yellow spectral regions; its wavelength dependence and position angle agrees with the proposed model.

The strong wavelength dependence of the position angle of polarization, similar to that observed for VY CMa, was found for the infrared objects NML Cyg and CIT 11 (KRUSZEWSKI 1971, DYCK, FORBES and SHAWL 1971); both of them are M-type supergiants and their dust envelopes may be very similar to that of VY CMa. The polarization of NML Cyg seems to be constant in the infrared but changing with time in the red spectral region where it reaches 17 per cent at $0.65 \mu$ (Fig. 17). It is probably extremely high at shorter wavelengths where the object is too faint for polarimetric observations.

\section{Suggestions and Plans for the Future Studies}

The weak point of the studies of intrinsic polarization so far is lack of the simultaneous polarimetric and spectrophotometric observations. The observations give us the changes in polarization, usually irregular, and we have nothing to correlate them with. We shall try to organize in Tucson the simultaneous polarimetry in visual region and infrared photometry in 3 to $11 \mu$ region, using different telescopes; this may be the best approach for deciding whether the same dust grains produce polarization and the infrared excess. Equally important would be the spectrophotometry in the 0.3 to $1 \mu$ region made simultaneously with polarimetry in the same spectral region. Having this in mind I designed for the University of Arizona an instrument which can be used both for spectrum scanning and for narrow-band polarimetry.

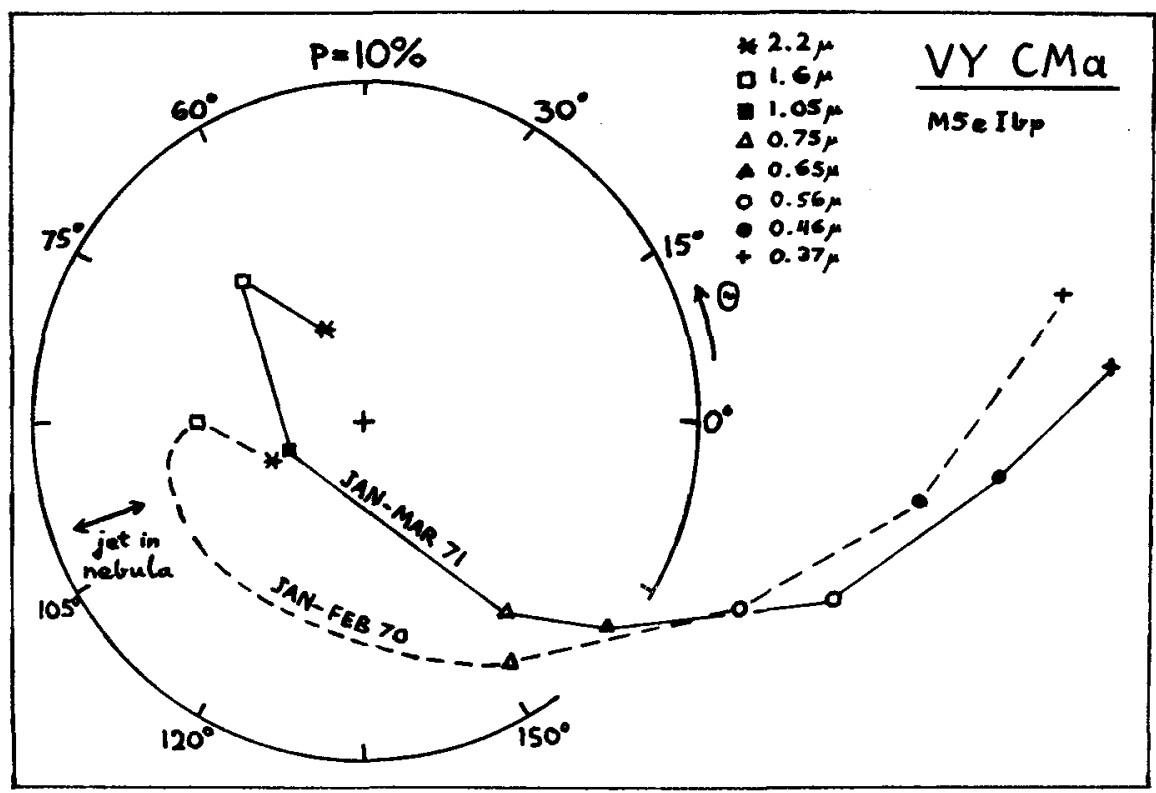

Fig. 16: Polarization of VY CMa plotted in radial coordinates. The lines join the observations made at different wavelengths approximately at the same time. 


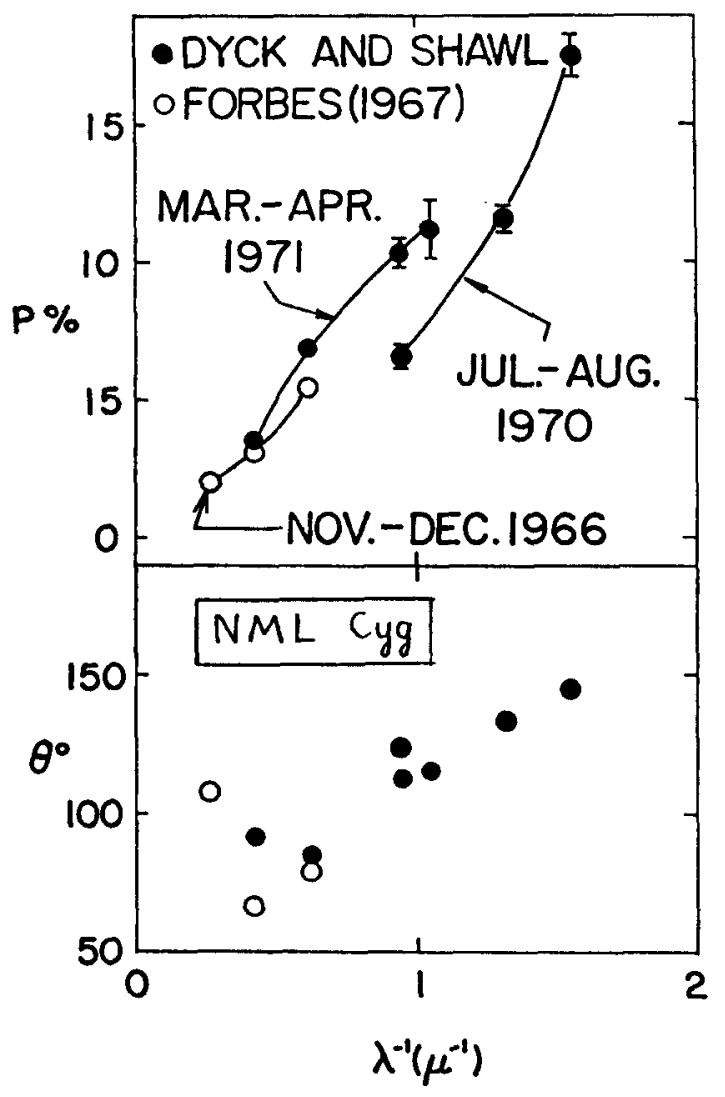

Fig. 17: Wavelength dependence of polarization for NML Cyg according to DYCK, FORBES and SHAWL (1971).

The essential component of the polarimetric part of this instrument is an achromatic half-wave plate, rapidly rotating in front of the Wollaston prism, as proposed by LYOT (1949). Two different methods of achromatizing are combined in the design of half-wave plate. The first method, discussed recently by BECKERS (1971), is using two different birefringent materials. I found that the most practical combination is a half-wave plate made of the plane parallel plate of crystallic quartz $0.304 \mathrm{~mm}$ thick and a plate of magnesium fluoride $0.262 \mathrm{~mm}$ thick. Such combination is a half-wave plate over a wider spectral region than a single halfwave plate, but still is far from perfection. The achromatism is considerably improved by using the method proposed by PANCHARATNAM (1955) and used in astronomical polarimetry by APPENZELLER (1967). In this method three half-wave plates are combined, the central one having its optical axis oriented differently than the other two. Using the quartz $+\mathrm{MgF}_{2}$ combination for each of the three constituent half-wave plates the deviations of retardation from half-wave (i. e. $180^{\circ}$ ) are negligible in the spectral range 0.3 to $1.0 \mu$. The PANCHARATNAM's device has a disadvantage that the position angle of its effective optical axis depends on wavelength (Fig. 18). Fortunately, this wavelength dependence can be eliminated by cementing in front of a Wollaston prism another PANCHARATNAM's half-wave plate, 
exactly identical to the rotating one. To avoid the motion of the image of telescope mirror on photocathodes, caused by nonplane-parallel shape of rotating half-wave plate, a lens $\mathrm{T}$ forms the image of telescope mirror on rotating half-wave plate.

Because of using the half-wave plates there is no need for the depolarizer. Therefore the mean errors of polarimetric results obtained in unit time are decreased by a factor of two; also, the narrow band filters can be used which is impossible with depolarizer. Our new polarimeter (Fig. 19) automatically cancels the polarization of sky background. Three adjacent focal plane diaphragms are used; the star is centered in the central one. The aforementioned lens
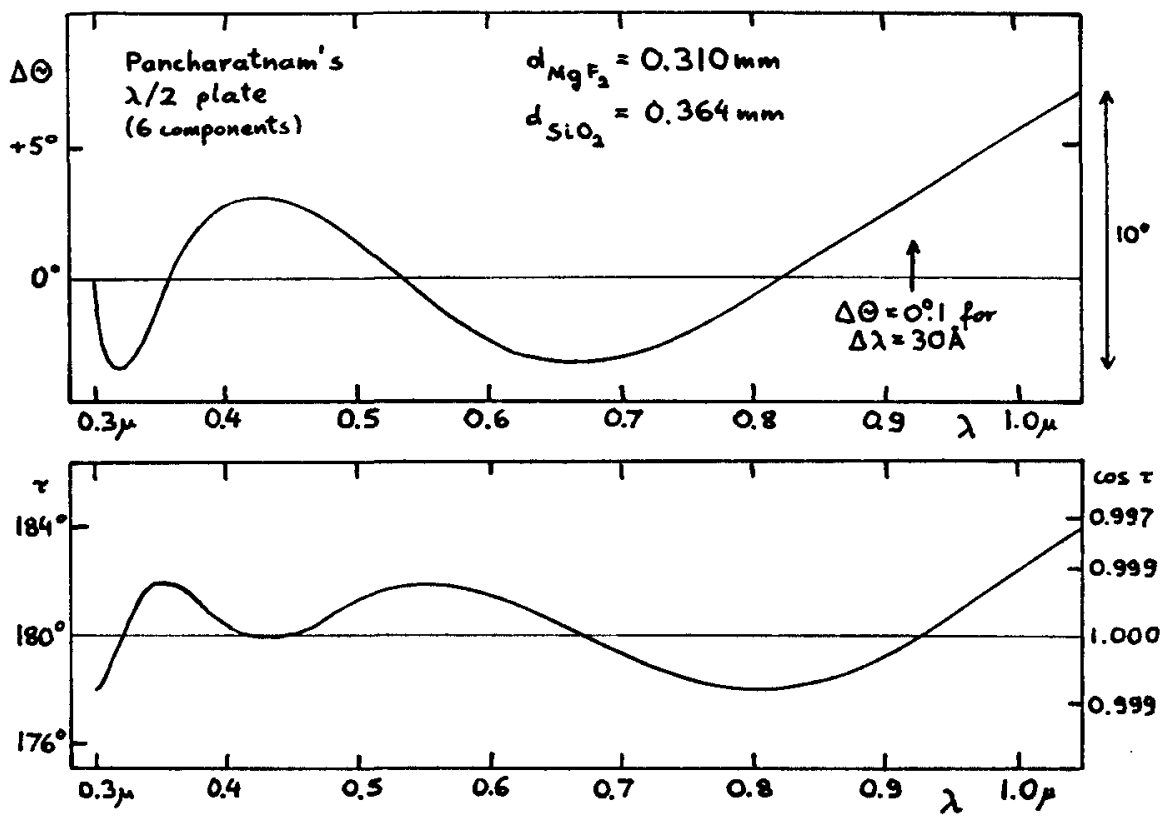

Fig. 18: Wavelength dependence of (a) position angle of effective optical axis, and (b) of the angular retardation of six-component PANCHARATNAM's half wave plate of quartz and magnesium fluoride.

T forms the images of diaphragms on two Fabry lenses. The ordinary image of the left diaphragm coincides with the extraordinary image of the central diaphragm so that the sky background in superimposed images becomes unpolarized; similarly, the extraordinary image of the right diaphragm cancels the polarization of sky background in the ordinary image of the central diaphragm.

The efficiency of the instrument is increased by using the dichroic filters which split the light into four spectral regions so that eight photomultipliers are used simultaneously. The ordinary and extraordinary beams are reflected from each of the dichroic filters under the same conditions because of somewhat unusual design of the Wollaston prism in which the crystalline optical axis of each component makes $45^{\circ}$ with the refracting edge of the prism. Therefore for each of the two beams the plane of polarization makes $45^{\circ}$ with the plane of incidence on the dichroic filter.

It is my hope that this new instrument will make possible a deeper insight into the phenomena responsible for the intrinsic polarization of variable stars.

I express my gratitude to the National Science Foundation for partial support of this work. 


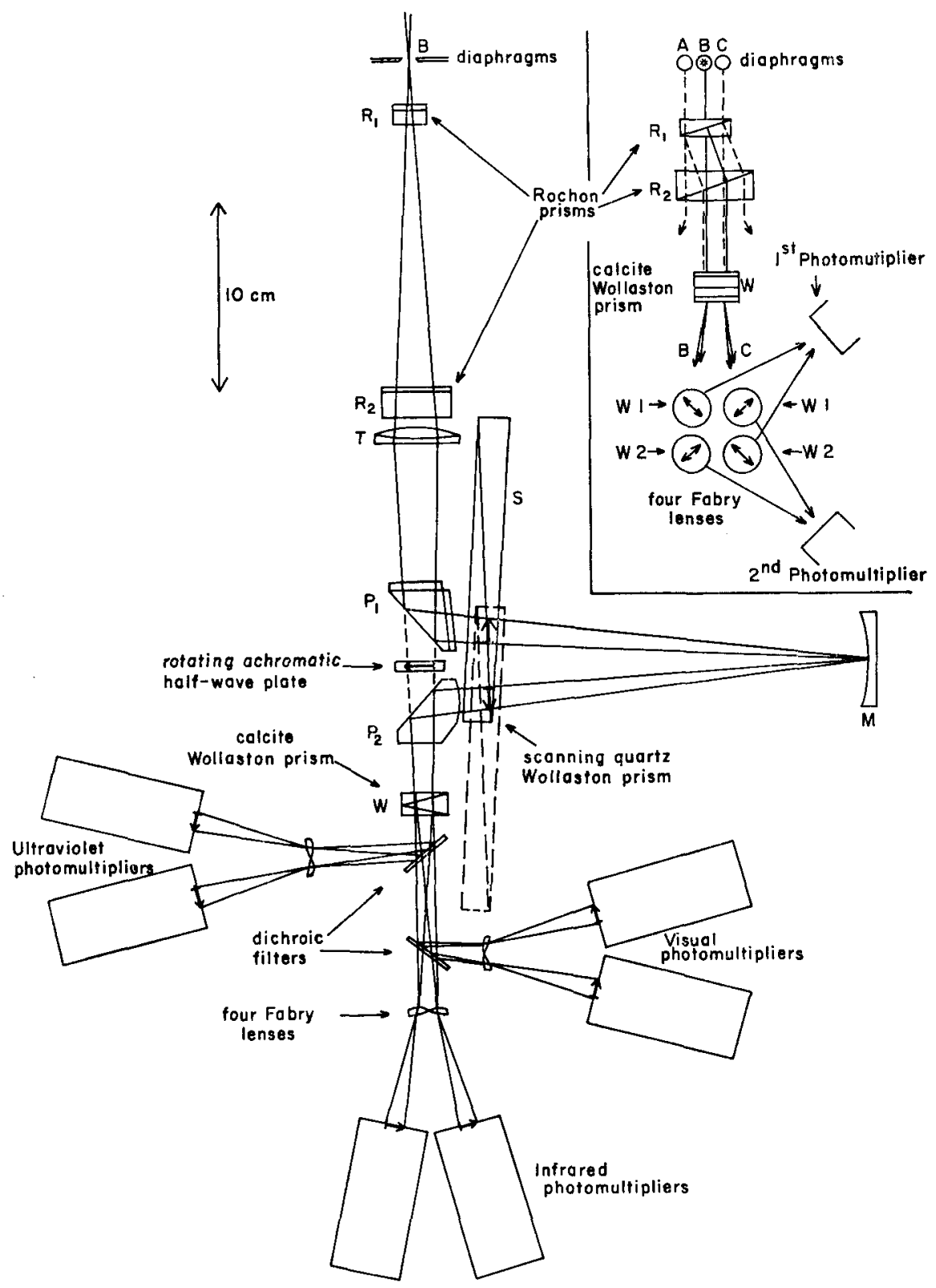

Fig. 19: Schematics of the polarimeter (description in the text). 
ALlEV, A. A., 1965, Astr. Circ. U.S. S. R., No. 337.

ANGEL, J. R. P., and LANDSTREEI, J. D. 1970, Astrophys. J. (Letters), 162, L61.

ANGEL, J. R. P., 1971, Astrophys. J. (Letters), 165, L71.

APPENZELLER, I., 1967, Publ. A. S. P., 79, 136.

APPENZELLER, I., and HILTNER, W. A., 1967, Astrophys. J., 149, 353.

BABCOCK, H. W., 1947, Astrophys. J., 105, 105.

BAHNG, J. D. R,, 1971, Astrophys. J. (Letiers), 167, L75.

BECKERS, J. M., 1971, Appl. Optics, 10, 973.

BEHR, A., 1959, Veröff. Göttingen Sternw., No. 126.

CLARKE, D., and GRAINGER, J. F., 1966, Ann. Ap. 29, 355.

COCKE, W. J., DISNEY, M. J., MUNCASTER, G. W., and GEHRELS, T. 1970, Nature, 227, 1327.

COLLINS, G. W., 1970, Astrophys. J. 159, 583.

COYNE, G. V., 1970a, Ric. Astr. Specola Vaticana, 8, 85.

COYNE, G. V., 1970b, idid., 8, 117 .

COYNE, G. V., 1971, in preparation.

COYNE, G. V., and GEHRELS, T., 1967, Astr. J., 72, 887.

COYNF, G. V., and A. KRUSZEWSKI, 1968, Astr. J., 73, 20.

COYNE, G. V., and A. KRUSZEWSKI, 1971, in preparation.

COYNE, G. Y., 1971, in preparation.

DOMBROWSKI J, V. A., 1970, Astrofiz. 6, 207

DONN, B., STECHER, T. P., WICKRAMASINGHE, N. C., and WILLIAMS, D. A., 1966, Astrophys. J., 145,949 .

DYCK, H. M., FORREST, W. J., GIILETT. F. C., STEIN, W. A., GEHRZ, R. D., WOOLF, N. J., and SHAWL, S. J., 1971, Astrophys. J., 165, 57.

DYCK, H. M., FORBES, F. F., and SHAWL, S. J., 1971, Astr. J., 76, 901.

DYCK, H. M., and JENNINGS, M. C., 1971, Astr. J., 76, 431.

DYCK, H. M., and SANDFORD, M. T., 1971, Astr. J., 76, 43.

EGGEN, O. J., MATHEWSON, D. S., and SERKOWSKI, K., 1967, Nature, 213, 1216.

ELVIUS, A., and ENGBERG, M., 1967, Arkiv för Astr., 4, 387.

FORBES, F. F., 1971, Astrophys. J. (Letters), 165, L83.

FRIEDEMANN, C., 1970, Mitt. Univ. Sternw. Jena, No. 104.

GEHRELS, T.. 1971, Vistas in Astronomy (in press)

GEHRZ, R. D., and WOOLF, N. J., 1970, Astrophys. J. (Letters), 161, L213.

GEHRZ, R. D., and WOOLF, N. J., 1971, Astrophys. J., 165, 285.

GEISEL, S. L., KLEINMANN, D. E., and LOW, F. J., 1970, Astrophys. J. (Letters) 161, L101.

GILIETT, F. C., MERRILL, K. M., and STEIN, W. A.,1971, Astrophys. J., 164, 83.

GRIGORYAN, K. A., 1958, Soob. Byurakan Obs., 25, 45.

GRIGORYAN, K. A., 1970, Soob. Byurakan Obs., 41, 13.

HALL, R. C., 1965, Publ. A.S.P., 77, 158.

HANNER, M. S., 1971, Astrophys. J., 164, 425.

HARRINGTON, J. P., 1970, Astroplys. Space Sc., 8, 227

IHARRINGTON, J. P., 1969, Astrophys. Let., 3, 165.

HASHIMOTO, J., MAIHARA, T., OKUDA, H., and SATO, S., 1970, Publ. Astr. Soc. Japan, $22,335$.

HILTNER, W. A., 1947, Astrophys. J., 106, 231.

HILTNER, W. A., and MOOK, D. E., 1966, Astrophys. J., 143, 1008.

HILTNER, W. A., and MOOK, D. E., 1967, Magnetic and Related Stars (R. C. Cameron, ed., Mono Book Corp.; Baitimore), p. 123 .

HILTNER, W. A., 1967, Magnetic and Related Stars (R. C. Cameron, ed., Mono Book Corp.; Baltimore), p. 123.

KEMP, J. C., 1970, Astrophys. J., 163, 169.

KEMP, J. C., SWENDIUUND, ]. B., LANDSTREET, J. D., and ANGEL, J. R. P., 1970, Astrophys. J., (Letters), 161, L77.

KEMP, J. C., and SWENDLUND, J. B., 1970, Astrophys. J., 162, L67.

KNACKE, R. F., GAUSTAD, J. E., GLLLETT, F. C., and STEIN, W. A., 1969, Astrophys. J. (Letters), 155, L189.

KRISHNA SWAMY, K. S., 1970, Astrophys. Space Sci., 9, 123.

KRUSZEWSKI, A., 1971, Astr. J., 76, 576.

KRUSZEWSKI, A., GEHRELS, T., and SERKOWSKI, K., 1968, Astr. J., 73, 677.

KRISTIAN, J., VISSVANATHAN, N., WESTPIIAL, J. A., and SNELLEN, G. H., 1970, Astrophys. J., $162,475$.

LANDSTREET, J. D., and ANGEL, J. R. P., 1971, Astrophys. J. (Letters), 165, L67.

LYOT, B., 1947, C. r., 226, 137.

MENDOZ̈A, E. E., 1967, Bull. Obs. Tonanzintla y Tacubaya, 4, 114.

NAGIRNER, D. I., 1962, Trudy Leningrad Astr. Obs., 19, 79.

NEUGEBAUER, G., BECKLIN, E., and HYLAND, A. R., 1971, Ann. Rev. Astr. Ap., 9. 67.

OHMAN, Y., 1934, Nature, 135, 534.

PANCHARATNAM, S., 1955, Proc., Indian Acad. Sci., A41, 137.

PENSTON, M. V., 1967, Observatory, 87, 276.

POLOSUKHIN $\Lambda$, N. C., 1969, Izv. Krim. Ap. Obs., 39, 34

RUCINSKI, S., 1066, Acta Astr., 16, 127.

RUCINSKI, S., 1967, ibid., 17, 207.

RUCINSKI, S., 1970, ibid,, 20, 1.

SCHMIDT, K. M., 1968, Astr. Nachr., 290, 271.

SER KOWSK I, K,, 1966, Astrophys. J., 144, 857.

SERKOWSKI, K., 1968, ibid., 154, 115.

SERKOWSKI, K., 1969a, Astrophys. J. (Letters), 156, L55.

SER KOWSK I, K., 1969b, ibid., 158, Lio7. 
SERKOWSKI, K., 1970a, Astrophys. J., 160, 1083.

SERKOWSKI, K., 1970b, ibid., 160, 1107.

SERKOWSKI, K., 1971, Contr. Kitt Peak Obs., No. 554, 107.

SERKOWSKI, K., and CHO JNACKI, W., 1969, Astr. Ap., 1, 442.

SERKOWSKI, K., and KRUSZEWSKI, A., 1969, Astrophys. J. (Letters), 155, L15.

SHAKHOVSKOJ, N. M., 1962, Astr. Zh., 39, 755 (Trans. Soviet Astr., 6, 587).

SHAKHOVSKO J, N. M., 1963, Astr. Zh., 40, 1055 (Trans. Soviet Astr., 7, 806).

SHAKHOVSKOJ, N. M., 1964, Astr. Zh., 41, 1042 (Trans. Soviet Astr., 8, 833).

SHAKHOVSKO J, N. M., 1969a, Astr. Zh., 46, 386 (Trans. Soviet Astr., 13, 303).

SHAKHOVSKO J, N. M., 1969b, Izv. Krim. Ap. Obs., 39, 11.

SHAWL, S. J., 1969, Astrophys. J. (Letters), 157, L57.

SHAWL, S. J., and ZELLNER, B., 1970, Astrophys, J. (Letters), 162, L19.

SHULOV, O. S., 1966, Astr. Circ. U.S.S. R., No. 385.

SHULOV', O. S., 1967, Trudy Leningrad Astr. Obs., 24, 38.

SHULOV, O. S., and GUDKOVA, G. A., 1969, Astrofiz., 5, 477.

STRITTMATTER, P. A., and SERKOWSKI, K., 1971, in preparation.

SVATOS, J., 1970, Bull. Astr. Inst. Czechosl., 21, 54.

THACKERAY, A. D., 1965, Obser, atory, 76, 154.

THIESSEN, G., 1961, Astr. Abh. Hamburg-Bergedorf, 5, No. 9.

VARDANIAN, R. A., 1964, Soob. Byurakan Obs., 35, 3.

VARDANIAN, R. A., 1966, ibid., 37, 23.

VARDANIAN, R. A., 1969, Astr. Circ. U. S.S. R., No. 508.

VARDANIAN, R. A., 1970, Astrofiz., 6, 77.

VISVANATHAN, N., 1967, M. N. R. A. S., 135, 275.

WALLERSTEIN, G., 1971, Astrophys. J., 166, 725.

WOOLF, N. J., and NEY, E. P., 1969, Astrophys. J. (Letters), 155, L181.

WOOLF, N. J., STEIN, W. A., and STRITIMATTER, P. A., 1970, Astr. Ap., 9, 252.

TELLNER, B., 1970, Astr. J., 75, 182.

ZELLNER, B., 1971, Astr. J., 76, 651.

ZELLNER, B., and MORRISON, N. D., 1971, Astr. J., 76, 645.

\section{Discussion to the paper of SERKOWSKI}

BAKOS: What are the instrumental limitations of polarization?

SERKOWSKI: For the fainter stars the accuracy of the polarimetry is limited only by the photon statistics. For example, the mean error of percentage polarization of a $12^{\text {th }}$ mag. star observed for 15 minutes with a 60 -inch telescope is about $\pm 0.25 \%$.

BEHR: According to our experiences at Göttingen and on La Silla the accuracy is practically given by the statistics of photoelectrons (if some instrumental limitations are observed). It is a question of time until the necessary amount of photons are counted, to get the accuracy which is wanted.

SAHADE: Are there enough observations of $\beta$ Lyr to tell whether there are variations depending on the cycle? This question is connected with another question I would like to. pose, namely, which is the accuracy of the percentage polarization observations?

SERKOWSKI: The polarimetric observations made till now are not accurate enough to detect the changes from cycle to cycle. The mean error of a single observation of $\beta \mathrm{Lyr}$ is about $\pm 0.05 \%$. It seems feasible now to make the observations with the mean errors 2 or 3 times smaller for a star as bright as $\beta$ Lyr.

SAHADE: How is it in the case of V $444 \mathrm{Cyg}$ ? When is the polarization of V444 Cyg larger? SERKOWSKI: The polarization is increasing during the eclipse.

SMAK: Are any observations planned for novae and $U$ Gem stars at their minima? These are really important for better understanding of disks.

SERKOWSKI: I am planning to make the polarimetric observations of some of these stars with the Steward Observatory 90-inch Telescope during the winter.

H.J. WOOD: Would you comment further for us on the polarization measurements of magnetic stars. In particular, such measurements were reported for HD 71866 and HD 215441 but not confirmed. Has any comprehensive survey of the magnetic variables been planned or carried out?

SERKOWSKI: None of the numerous series of wide-band polarimetric observations of magnetic stars gave the convincing evidence of the existence of intrinsic polarization. Such polarization must be very small and $I$ doubt if at the present polarimetric accuracy the further surveys would be worthwhile. 